\title{
Síndrome de Münchausen por poder en otorrinolaringología
}

\author{
Münchausen syndrome by proxy in otorhinolaryngology
}

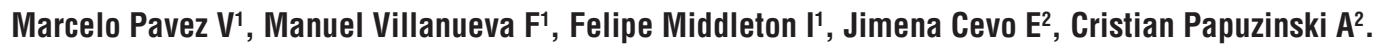

\begin{abstract}
RESUMEN
El síndrome de Münchausen por poder es una forma de maltrato infantil, con graves consecuencias para el niño afectado. El diagnóstico de esta afección constituye un desafío para los médicos por su heterogeneidad en los síntomas y signos de presentación. Variadas manifestaciones otorrinolaringológicas están descritas en esta patología. En este artículo se presenta un caso clínico de otitis media crónica con otorragia en un paciente de 2 años, donde se logró establecer el diagnóstico de síndrome de Münchausen por poder a través de la identificación de elementos incongruentes en la historia clínica y finalmente por la detección in fraganti de la agresión por parte de la madre. En esta revisión se concluye que es necesario un alto índice de sospecha para identificar esta patología, la que debe ser una preocupación habitual del equipo de salud para evitar asi consecuencias graves en estos pacientes, tales como secuelas psiquiátricas o incluso la muerte.
\end{abstract}

Palabras clave: Síndrome de Münchausen por poder, otitis media crónica, otorragia.

\begin{abstract}
Münchausen's syndrome by proxy is a form of child abuse with severe consequences for the affected child. The diagnosis of this condition is a challenge for physicians due to the heterogeneity of the presentation symptoms. A lot of otorhinolaryngological manifestations have been described in this syndrome. We present a case of a 2 year old patient with chronic otitis media and otorrhagia, where we could establish the diagnosis of Münchausen's syndrome by proxy through the identifications of incongruent elements on the clinical history and finally, the direct visualization of mother's aggression. In this should be a common concern for health workers to avoid severe consequences in these patients like psychiatric diseases or even death.
\end{abstract}

Key words: Münchausen's syndrome by proxy, chronic otitis media, bleeding ears.

\footnotetext{
1 Interno de Medicina, Universidad de Valparaíso.

2 Médico Cirujano, Servicio de Otorrinolaringología, Hospital Carlos Van Buren, Universidad de Valparaíso.
}

Recibido el 28 de marzo, 2016. Aceptado el 10 de abril, 2016. 


\section{INTRODUCCIÓN}

El síndrome de Münchausen tiene como características la fabricación de síntomas y signos de una enfermedad, cuyo objetivo es la búsqueda de ayuda, compasión y atención médica por parte del paciente ${ }^{1,2}$. La expresión en inglés "Münchausen by proxy", o Münchausen por poderes en español (SMPP) fue acuñado por primera vez por Roy Meadow ${ }^{3}$ en 1977, haciendo referencia a la situación en que los padres o cuidadores, la mayoría de veces la madre, simulan síntomas o causan enfermedad en un niño, llevando a evaluaciones y tratamientos innecesarios ${ }^{4}$.

El SMPP constituye una forma de maltrato infantil, de difícil diagnóstico, que a menudo pasa desapercibido durante semanas, meses e incluso años, y que puede significar graves complicaciones e incluso la muerte para el pequeño ${ }^{5}$.

Una presentación muy frecuente de SMPP en los niños pequeños es la simulación de eventos que aparentemente amenazan la vida o ALTE (apparent life threatening events), que suelen presentarse como episodios de apneas y convulsiones muy inespecíficos, y que deberían llevarnos a la sospecha de maltrato ${ }^{6}$.

La otorrinolaringología no queda ajena a esta condición, ya que se han descritos muchas manifestaciones clínicas, la mayoría inespecíficas y poco claras. Por ello estar consciente de la existencia de este síndrome, es el primer paso para la sospecha, diagnóstico y posterior resolución de estos casos ${ }^{4}$.

A continuación se presenta un caso clínico ocurrido en el Servicio de Pediatría del Hospital Carlos Van Buren (HCVB) de Valparaíso, que ejemplifica la importancia de incluir esta patología dentro de nuestro arsenal diagnóstico.

\section{CASO CLÍNICO}

Paciente de 2 años 9 meses, con antecedentes de síndrome bronquial obstructivo recurrente y retraso del desarrollo psicomotor de causa no precisada, consulta en reiteradas ocasiones en el Hospital de San Antonio por cuadro de otalgia bilateral con otorrea y otorragia intermitente.

Su primera consulta fue a los 2 años de edad, donde se hizo diagnóstico de otitis media aguda bilateral y se manejó con tratamiento antibiótico estándar con evolución favorable. Posterior a eso, se repitieron múltiples consultas por la misma causa siendo hospitalizado en varias ocasiones, donde recibió diferentes esquemas antibióticos, y se aislaron diversos agentes en cultivos de secreción ótica, destacando patógenos poco habituales para otitis media en etapa preescolar.

Por su tórpida evolución fue derivado al Servicio de Pediatría del Hospital Carlos Van Buren, donde fue atendido en conjunto con el equipo de otorrinolaringología e infectología infantil. Al examen otorrinolaringológico se encontró en varias oportunidades otorrea bilateral, granulomas en los conductos auditivos externos que impedían ver los tímpanos, siendo diagnosticado como otitis media crónica. Se realizó estudio microbiológico de la otorrea, donde se encontraron en distintos cultivos Candida guilliermondii, Escherichia coli y Staphilococcus aureus meticilino resistente; dado esos hallazgos, el niño recibió tratamientos antibióticos con cefotaxima, amoxicilina con ácido clavulánico, claritromicina, coprofloxacino ótico, clotrimazol, vancomicina y clindamicina en distintas oportunidades. Durante el periodo de hospitalización para los tratamientos antibióticos, junto con curaciones de oído frecuentes, el niño presentó una resolución momentánea del cuadro, pero que recurría una vez que era dado de alta.

Debido a las características particulares de la enfermedad y a las lesiones granulomatosas encontradas en los conductos auditivos externos, se decidió realizar biopsias, las que fueron informadas como exudado fibrinoleucocitario (Figura 1). Después de alrededor de 2 meses, se sospecha que la madre podría estar instilando alguna sustancia irritante en los oídos del niño, situación evaluada de manera conjunta con asistente social y psiquiata infantil, siendo desestimada en primera instancia tras el interrogatorio.

A lo largo de estos nueve meses trascurridos desde la primera consulta, el paciente presentó 5 hospitalizaciones, sumando un total de 83 días cama al momento de la última consulta, siempre por el mismo cuadro clínico.

En enero de 2016, el paciente fue derivado nuevamente al Servicio de Pediatría del Hospital Carlos Van Buren por recurrencia de su patología. Durante esta hospitalización, un tercero sorprendió 


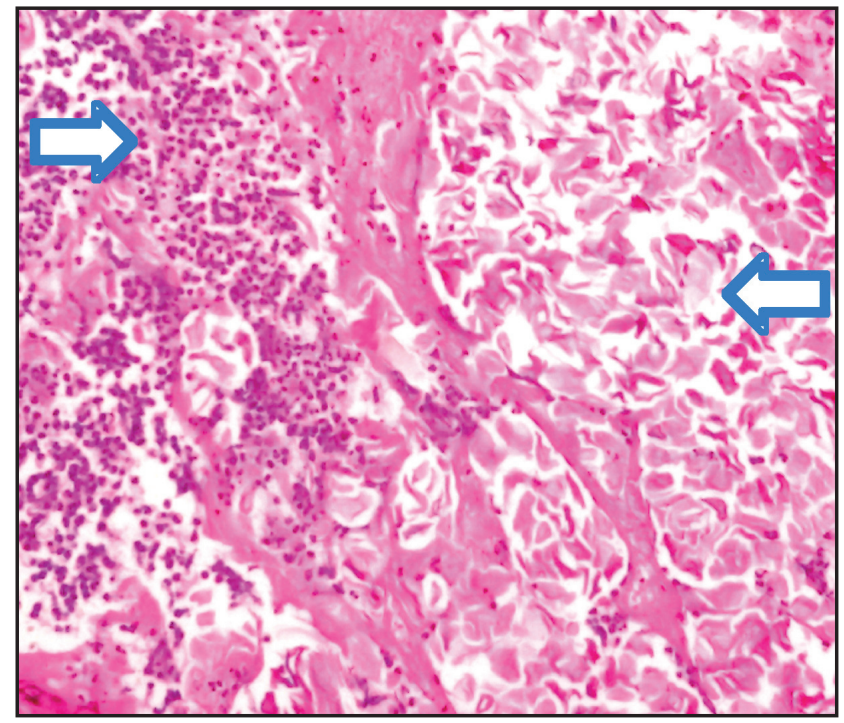

Figura 1.

a la madre del paciente administrándole algún medicamento por vía endovenosa -aparentemente dipirona- en dos ocasiones a su hijo, sin indicación médica. En esa oportunidad, la madre amenazó para que guardara silencio a quien la había observado, por lo que se dio aviso al personal de turno. Posterior a la demanda correspondiente, se encontraron en las ropas de la madre la jeringa que habría utilizado para la administración del fármaco y bajo las sábanas de la cama una aguja no perteneciente al arsenal del hospital. Al examen otológico tras este incidente, se hallaron nuevas lesiones en ambos conductos auditivos externos, concordantes con traumatismos recientes. Dada aquella situación, en el contexto de la investigación a cargo del Tribunal, se ordena la prohibición del ingreso de la madre al recinto hospitalario.

Tras la separación de la madre, el paciente evoluciona favorablemente, manteniendo controles frecuentes en otorrinolaringología. El niño es dado de alta en buenas condiciones generales, sin lesiones en los conductos auditivos externos y por primera vez se logran ver sus tímpanos, los cuales afortunadamente se encuentran sin alteraciones. Inclusive destaca una mejoría sustantiva de la interacción del niño con otras personas. Queda a discreción del Tribunal la conducta legal a seguir.

\section{DISCUSIÓN}

En el síndrome de Münchausen por poder, el cuidador finge o provoca síntomas a una tercera persona, el cual suele ser un niño. La motivación, como en otros trastornos ficticios, es asumir el rol de enfermo, en este caso sin ganancia secundaria directa, como sería en el caso del síndrome Münchausen (no por poderes), en donde es el paciente el que persigue algún beneficio al asumir el rol de enfermo?.

Se desconoce la verdadera incidencia de los casos de SMPP, principalmente porque es un síndrome poco sospechado, y por consecuencia subdiagnosticado. La madre es la abusadora en el $75 \%$ de los casos. No existe diferencias por género de los niños maltratados, tardando en promedio 2 años para el diagnóstico desde la primera consulta y con un promedio de 20 consultas antes de precisar el diagnóstico ${ }^{5}$.

Respecto a las manifestaciones otorrinolaringológicas de esta patología solo existe un caso reportado en Chile, tratándose de un cuadro de otalgia asociado a intoxicación por clorpromazina ${ }^{8}$.

En la literatura occidental hay varios casos de SMPP con otorragia, dentro de los cuales desta- 
can: esputos sanguinolentos que la madre escupía en el oído de su hijo ${ }^{9}$, otorragia secundaria a traumatismos repetitivos infligidos por la madre ${ }^{4}$ y un caso de otorragia secundaria a la administración de anticoagulantes sin indicación médica también por parte de la madre ${ }^{10}$.

Las características que deben hacer sospechar de un caso de SMPP son ${ }^{11-13}$ :

a) La enfermedad es generalmente multisistémica, prolongada, recurrente, e inexplicable; b) Los síntomas son incongruentes y no tienen sentido clínico; c) Los resultados de exámenes no se condicen con la situación del niño; d) Los síntomas y signos no están presentes en ausencia de los padres; e) Uno de los padres está muy unido al niño, generalmente la madre, y el otro, está ausente durante la hospitalización; f) Los padres muestran gran preocupación por los sentimientos del personal médico; g) Los tratamientos indicados son poco tolerados, por ejemplo los vendajes y las vías venosas fallan; $h$ ) Las convulsiones se dan sin testigos y no responden a tratamiento anticonvulsivante $^{4}$.

Ciertamente, estas características no son una pauta rígida para reconocer el SMPP, sino más bien pueden ser útiles a los clínicos y al personal para llegar a la sospecha y poder establecer este tipo de diagnóstico, habiendo descartado previamente que las manifestaciones no sean secundarias a alguna condición médica que se haya ignorado.

Es necesario ser muy cuidadoso con la sospecha y correcto diagnóstico de este síndrome. Considerando que una acusación equivocada llevará a la separación del niño de sus padres, pudiendo llegar incluso a la pena de cárcel de estos últimos. Existen casos en que se han hecho acusaciones no bien fundamentadas y donde finalmente el personal médico ha manipulado información para lograr la captura y separación de los padres. El mismo Meadow fue privado de su facultad para ejercer medicina luego que se comprobara la inocencia de padres condenados a pena de cárcel, contra los que él había dado falsos testimonios siendo perito de los casos investigados.

Se debe considerar que al momento de sospechar o estar frente a un caso de Münchausen por poder, el testigo sólo debe proporcionar información objetiva e imparcial, siempre presentando evidencia real y nunca modificándola. En caso de ser médico y ser perito en un caso, sólo se debe atestiguar en áreas en las que se es experto, y nunca tomar el rol de acusador o defensor, se debe ser siempre imparcial, esto ayudará a la correcta resolución del caso y llevará menos probablemente a una equivocada resolución ${ }^{14}$.

En el caso expuesto, la madre del paciente siempre mostró preocupación por la condición médica de su hijo, en cambio el padre se mantuvo ausente durante toda la hospitalización. El principal elemento de sospecha consistió en la ausencia de una causa clara para las constantes recurrencias de nuestro paciente, sumado a la clara mejoría en el cuadro clínico luego de que el paciente fuera apartado de su entorno habitual, y por ende, del maltrato propinado por su madre. Otros elementos de sospecha fueron las características clínicas infrecuentes de su enfermedad, tales como aislamiento de patógenos poco habituales en infecciones del oído medio y lesiones granulomatosas en el conducto auditivo externo al examen.

El SMPP constituye una enfermedad grave, con una mortalidad de alrededor de $7 \%$, y un alto porcentaje de secuelas psiquiátricas en las víctimas, donde destacan los trastornos de estrés postraumático, depresión y ansiedad ${ }^{15}$.

A raíz de estas consecuencias, nos parece que es rol del equipo de salud velar por la correcta identificación de todas las formas de maltrato infantil, teniendo en cuenta que muchas veces las manifestaciones de esta condición pueden simular otros cuadros clínicos como ocurre en el SMPP. Un alto índice de sospecha será necesario para la identificación precoz de esta patología, ya que es probable que en la actualidad se esté subdiagnosticando ${ }^{12}$.

\section{CONCLUSIONES}

Las manifestaciones del SMPP son múltiples e inespecíficas, pudiendo afectar cualquier sistema u órgano. El compromiso otológico en esta enfermedad está descrito en varios casos de la literatura mundial, sin embargo, pareciera ser un diagnóstico poco frecuente según la literatura chilena. Es necesario un alto índice de sospecha por parte de todo el equipo de salud para realizar el diagnóstico de esta patología. La identificación 
tardía de este síndrome puede traer consecuencias fatales para las personas maltratadas, además de múltiples secuelas biopsicosociales en las víctimas, donde destacan trastornos de la esfera psiquiátrica, tales como estrés postraumático, ansiedad patológica y depresión. El personal de salud deberá asumir una postura activa en la búsqueda y resolución de esta forma de maltrato, para proteger a la población vulnerable, especialmente pediátrica, teniendo especial cuidado en no caer en acusaciones falsas o infundadas, y siendo siempre objetivo e imparcial a la hora de evaluar un posible caso de Münchausen por poderes.

\section{BIBLIOGRAFÍA}

1. Sussman N, Hyler S. Factitious Disorders. En: Comprehensive Textbook of Psychiatry IV, de Kaplan H y Sadock B. Williams y Wilkins Editores. Baltimore, 1985.

2. American Academy of Family Physicians, American Psychiatric Association. Work Group on DSM-IVPC. Diagnostic and statistical manual of mental disorders, fourth edition : primary care version. $1^{\text {st }}$ ed. Washington, DC: American Psychiatric Association; 1995. xv, 223 p. p.

3. Meadow R. Münchausen syndrome by proxy the hinterland of child abuse. Lancet 1977; 2(8033): 343-5.

4. Griffiths H, Cuddihy PJ, Marnane C. Bleeding ears: a case of Münchausen syndrome by proxy. International Journal of Pediatric Otorhinolaryngology 2001; 57(3): 245-7. PubMed PMID: 11223457.

5. Goni González T, Martínez Roda MJ, de la Cerda Ojeda F, Gómez de Terreros I. [Münchausen syndrome by proxy]. Anales de Pediatria 2008; 68(6): 609-11. PubMed PMID: 18559202. Sindrome de Münchausen por poderes.
6. Maida S. Ana Margarita MPME, Carrasco Ch. XIMENA. Síndrome de Münchausen-por-poder: un diagnóstico a considerar. Rev Chil Pediatr [Internet] 1999; 70(3): 215-20.

7. Landa-Contreras Ernesto A-AMP, Fortes-Álvarez José L. Síndrome de Münchausen por poderes: presentación de un caso y revisión de la literatura. Rev Asoc Esp Neuropsiq [revista en la Internet] 2014; 34(124): 791-5.

8. Madrid R GR, Pacheco $S$, Jaramillo Y. Resúmenes Del XLVII Congreso Chileno Pediatría. Noviembre 2007, Antofagasta - Chile. Revista Chilena de Pediatría 2007; 78: 623-84.

9. Bennett AM, Bennett SM, Prinsley PR, Wickstead M. Spitting in the ear: a falsified disease using video evidence. The Journal of Laryngology and Otology 2005; 119(11): 926-7. PubMed PMID: 16354350.

10. White ST, Voter K, Perry J. Surreptitious warfarin ingestion. Child Abuse \& Neglect 1985; 9(3): 349-52. PubMed PMID: 4052840.

11. Leonard KF, Farrell PA. Münchausen's syndrome by proxy. A little-known type of abuse. Postgraduate Medicine 1992; 91(5): 197-204. PubMed PMID: 1561159.

12. Meadow R. Münchausen syndrome by proxy. Archives of Disease in Childhood 1982; 57(2): 92-8.

13. DiBiase P, Timmis H, Bonilla JA, Szeremeta W, Post JC. Münchausen syndrome by proxy complicating ear surgery. Archives of Otolaryngology--Head \& Neck Surgery 1996; 122(12): 1377-80. PubMed PMID: 8956754.

14. Fitzpatrick M. Roy Meadow: the GMC's shame. The British Journal of General Practice 2005; 55(517): 647.

15. Bools CN NB, Meadow SR. Follow up of victims of fabricated illness (Münchausen syndrome by proxy). Arch Dis Child 1993; 69(6): 62530. 